\title{
Flore spontanée d'Algérie : différenciation écogéographique des espèces et polyploïdie
}

\author{
Rachid Amirouche ${ }^{1}$ \\ Marie-Thérèse Misset ${ }^{2}$ \\ ${ }^{1}$ Faculté des sciences biologiques, \\ Laboratoire de biologie et physiologie \\ des organismes (LBPO) \\ Équipe Biosystématique, \\ génétique et évolution \\ Université des sciences et de la technologie \\ Houari Boumediene (USTHB) \\ 32 El-Alia, Bab-Ezzouar \\ 16111, Alger \\ Algérie \\ <ramirouche@hotmail.com> \\ ${ }^{2}$ Université de Rennes 1UMR 6553 \\ EcoBioÉquipe "Mécanismes à l'Origine \\ de la Biodiversité " \\ Campus de Beaulieu, \\ Bât. 14A35042 Rennes cedex \\ <marie-therese.misset@univ-rennes1.fr>
}

\begin{abstract}
Résumé
En Algérie, les recherches nouvelles de botanique systématique, phylogénétique et phytogéographique, sont rares. Dans cette mise au point, nous soulignerons l'importance des études systématiques au niveau des espèces et des populations dans l'interprétation de la structure et de la dynamique de la flore spontanée algérienne, ainsi que leurs impacts sur l'évaluation et la conservation de la biodiversité. Les résultats illustratifs sont issus d'études sur quelques modèles de graminées (Poaceae) annuelles et pérennes, de légumineuses (Fabaceae) et de plantes à bulbes de la famille des Hyacinthaceae. Ils sont présentés dans un contexte de mise à jour taxonomique et de compréhension de la flore de l'Algérie et mis en rapport avec le rôle majeur des conditions bioclimatiques sur l'organisation de la diversité génétique des espèces. Bien que partiels, ces résultats mettent en relief : 1) la distribution géographique particulière des populations et taxons diploïdes et polyploïdes, et donc le rôle de la polyplö̈die dans les phénomènes de rareté et d'isolement ; 2) l'importance des zones de transition bioclimatique et des zones marginales, situées à la périphérie de l'aire de répartition, où l'existence de populations isolées favorise l'émergence de génotypes adaptés. Ces études ont une valeur prédictive pour l'inventaire des taxons et des populations spontanées qui doivent en priorité s'intégrer, tant dans les programmes de valorisation des ressources phytogénétiques que dans la stratégie de conservation de la biodiversité.
\end{abstract}

Mots clés : Algérie ; biodiversité ; facteur du milieu ; polyploïdie ; ressource génétique végétale.

Thèmes : ressources naturelles et environnement ; productions végétales.

\section{Abstract \\ Spontaneous flora of Algeria: Ecogeographical differentiation of species and polyploidy}

In Algeria, new research in systematic botany, phylogenetics and phytogeography is rare. In this paper, we underline the importance of systematic studies on species and population levels to interpret the structure and dynamic of spontaneous Algerian flora and its impact on the evaluation and the conservation of biodiversity. The results are from studies on annual and perennial graminaceous (Poaceae), leguminous (Fabaceae) species and Hyacinthaceae. Results are presented in a context of the taxonomic update and comprehension of Algerian flora and in relation with the major role of the bioclimatic conditions on species genetic diversity. These results highlight: (1) the particular geographical distribution of taxa and diploids and polyploid populations and the role of polyploidy in the phenomena of scarcity and isolation, (2) the importance of the transitional bioclimatic area and the marginal zones located at the periphery of the distribution area, where the isolated populations produce adapted genotypes. These studies have a predictive value for the inventory of taxa and populations which must be integrated in priority in the programmes of valorization of the phytogenetic resources and in the strategy of conservation of the biodiversity.

Key words: Algeria; biodiversity; environmental factors; plant genetic resources; polyploidy.

Subjects: natural resources and environment; vegetal productions.

Tirés à part : R. Amirouche 
a région méditerranéenne abrite une diversité biologique de première importance. La plupart des espèces de la flore spontanée, en Afrique du Nord notamment, sont remarquablement résistantes et bien adaptées à la sécheresse et à la salinité et constituent une part non négligeable des ressources génétiques locales à valeurs pastorale, fourragère, alimentaire, aromatique et médicinale (Abdelguerfi et Laouar, 1999; Ohba et Amirouche, 2003).

En raison de sa situation particulière en région méditerranéenne et de l'impressionnant gradient bioclimatique Nord-
Sud qui la caractérise (figure 1), l'Algérie offre des opportunités exceptionnelles pour l'évaluation et pour la compréhension des mécanismes impliqués dans la diversification et l'adaptation des plantes en relation avec l'évolution de leur environnement. En Algérie tellienne, les secteurs humides et subhumides comportent des "hotspots" ou points chauds de biodiversité unique (Médail et Quézel, 1997 ; Quézel et Médail, 2003 ; Véla et Benhouhou, 2007). Dans les zones de transition biogéographique, les fluctuations des conditions écologiques et l'hétérogénéité des habitats sont les facteurs déterminants de la richesse floristique et de la diversité génétique.

La flore est aujourd'hui très sérieusement menacée, en raison de la forte régression des milieux naturels sous l'action de l'homme, mais aussi parce que cette région serait l'une des plus exposées aux changements climatiques globaux (Sala et al., 2000 ; Hoekstra et al., 2005 ; Médail et Quézel, 2005).

En Algérie, les efforts de préservation sont surtout orientés vers la mise en ouvre d'aires protégées (Parcs nationaux, réserves naturelles) principalement situées

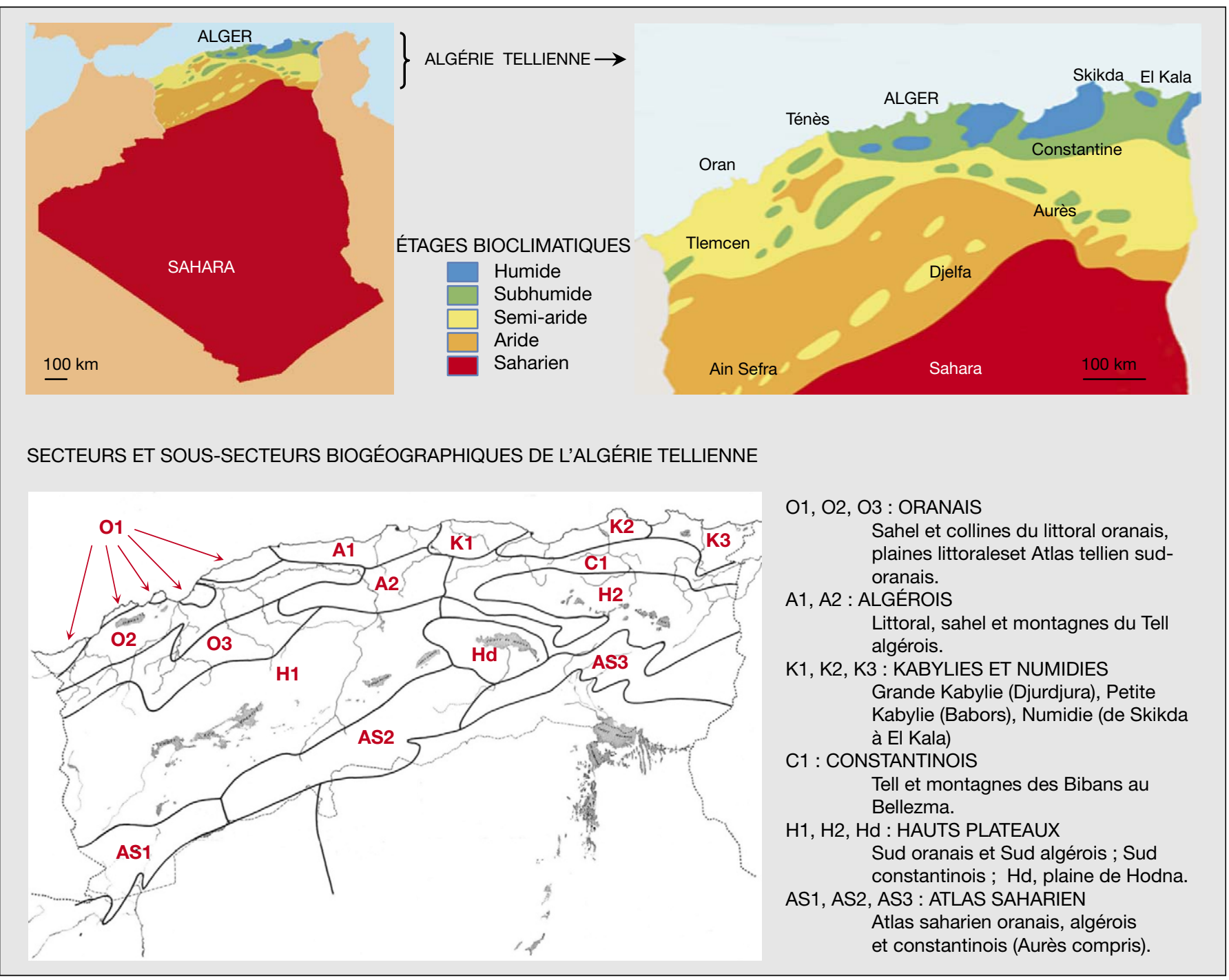

Figure 1. Contexte bioclimatique et biogéographique de l'Algérie.

Figure 1. Bioclimatic and biogeographic context of Algeria.

Les limites des étages bioclimatiques ont été établies d'après la carte bioclimatique simplifiée de Stewart (1974). Elles sont définies par trois paramètres principaux du climat méditerranéen : la pluviosité annuelle $(\mathrm{P})$, la moyenne des températures du mois le plus chaud $\left(\mathrm{M}{ }^{\circ} \mathrm{C}\right)$ et la moyenne des températures du mois le plus froid $\left(\mathrm{m}{ }^{\circ} \mathrm{C}\right)$. La carte de répartition des secteurs biogéographiques est celle de Quézel et Santa (1962). 
dans les écosystèmes du Tell et correspondant aux "botspots" connus de biodiversité. La gestion de ces milieux et les stratégies de conservation sont actuellement limitées par une connaissance insuffisante des unités biologiques en présence, réduite aux travaux botaniques classiques devant être mis à jour (encadré 1), et des mécanismes gouvernant leur évolution.

Les programmes de recherche des institutions et des équipes universitaires consacrés aux thèmes de la végétation sont essentiellement dédiés aux écosystèmes forestiers et steppiques et à un degré moindre aux zones arides (Médiouni, 2004). Les zones de transition qui joueraient un rôle essentiel dans la diversité génétique et le potentiel évolutif des espèces (Araújo, 2002), restent négligées. Au niveau spécifique, les données récentes relatives aux espèces spontanées à valeur patrimoniale sont encore peu nombreuses et les données relatives aux espèces rares ou endémiques sont quasiment inexistantes.

Les études pluridisciplinaires réalisées ces dernières années par différents laboratoires et équipes universitaires s'inscrivent dans une perspective de caractérisation des espèces spontanées à valeur agronomique, à la lumière notamment des données moléculaires dans les populations naturelles provenant de différents milieux. Elles ont jeté les bases d'un inventaire indispensable des espèces et des populations, de la connaissance de leur aire de répartition géographique, de leurs caractéristiques phénologiques et écophysiologiques et de leur vulnérabilité face aux pressions anthropiques et aux changements climatiques.

Nous exposerons ici des résultats obtenus sur des Poaceae, des légumineuses (Fabaceae) et des plantes à bulbes de la famille des Hyacinthaceae. Les analyses pluridisciplinaires morphologiques, cytogénétiques, biochimiques et moléculaires, appliquées à un nombre important de populations échantillonnées dans diverses conditions écologiques, visent à appréhender la variabilité génétique intra- et interpopulations et sa structuration écogéographique.

L'accent sera mis, d'une part, sur le rôle de la polyploïdie, une des principales sources d'adaptation, d'évolution et de spéciation chez les végétaux (Stebbins, 1971), et, d'autre part, sur la nécessité de révisions taxonomiques de notre flore.

\section{Complexes polyploïdes de Poaceae}

Avec plus de 320 espèces et sous-espèces, les Poaceae représentent l'une des plus importantes familles de la flore algérienne, famille remarquable en taxons rares et très rares $(\sim 38,5 \%)$ et en endémiques au sens large $(\sim 7,8 \%)$ - données établies à partir de la flore de Quézel et Santa (1962). Nous évoquerons, pour cette famille, le cas de complexes polyplö̈des d'espèces annuelles (Hordeum, Aegilops) et pérennes (Dactylis, Lygeum). Ces espèces constituent souvent la composante essentielle des pâturages, des prairies et des jachères. Elles sont caractérisées par une importante variabilité génétique et une tendance forte à une structuration des populations en fonction des conditions écogéographiques.

\section{Orges (Hordeum)}

Les orges spontanées du complexe Hordeum murinum L. sensu lato, sont représentées par deux taxons, l'un diploïde $(2 \mathrm{n}=2 \mathrm{x}=14), H$. murinum ssp. glaucum (Steudel) Tzvelev, l'autre tétraploïde $(2 \mathrm{n}=4 \mathrm{x}=28), H$. murinum ssp. leporinum (Link) Arg. Des analyses pluridisciplinaires ont montré une diversité génétique considérable des populations et une distribution géographique des cytotypes $2 \mathrm{x}$ et $4 \mathrm{x}$ fortement corrélée au gradient bioclimatique Nord-Sud (Amirouche et Misset, 2003).

Les populations tétraploïdes de la sousespèce leporinum sont très polymorphes

\section{Encadré 1 \\ Bref historique des recherches botaniques en Algérie (d'après Alcaraz, 1976)}

Les premières recherches botaniques en Algérie ont été menées dès la fin du XVIII ${ }^{e}$ siècle et nous devons les premiers inventaires et descriptions à Jean-Louis Marie Poiret (Poiret, 1789) avec son Voyage en Barbarie et à René Louiche Desfontaines avec Flora Atlantica (Desfontaines, 1798-1799).

Dès 1837, la "recherche botanique officielle " et les multiples missions d'exploration scientifique de l'Algérie, ont permis à de nombreux botanistes de constituer les premières collections et les premiers herbiers. Ceux d'Ernest SaintCharles Cosson et Michel Charles Durieu De Maisonneuve (1854-1867) sont parmi les plus illustres.

Les premières véritables flores, qui font encore référence aujourd'hui, sont celles de Jules Aimé Battandier et Louis Trabut, dont Flore d'Alger et catalogue de plantes d'Algérie (Battandier et Trabut, 1884) et Flore analytique et synoptique de l'Algérie et de Tunisie (Battandier et Trabut, 1902).

Mais c'est à René Maire (Maire, 1955-1987) que I'on doit une flore exhaustive, la célèbre Flore de I'Afrique du Nord dont les nombreux volumes (1 à 16) ont été publiés à titre posthume à partir de 1953.

Pierre Quezel et Sébastien Santa (Quézl et Santa, 1962-1963) ont proposé, avec leur Nouvelle flore de l'Algérie et des régions désertiques méridionales, un ouvrage plus "simple " qui constitue, de nos jours encore, la seule référence " accessible» sur la flore de l'Algérie.

En dépit du renouveau de la botanique systématique suscité à la faveur de données moléculaires (APG II, 2003), aucune révision taxonomique majeure n'a été entreprise, sinon des mises à jour de nomenclature et de synonymie, au demeurant très insuffisantes. Nous citerons entre autre, I'Index synonymique et bibliographique de la flore d'Afrique du Nord en cours d'élaboration par Alain Dobignard (Dobignard, 2009 [sous presse]).

Cependant, dans de nombreux cas, les déterminations sont rendues délicates en raison d'une complexité biologique qui n'est pas prise en compte (plasticité phénotypique, hybridation, polyploïdie). En effet, un grand nombre de taxons montrent un polymorphisme important et, parfois, constituent des complexes spécifiques aux délimitations taxonomiques peu évidentes. 


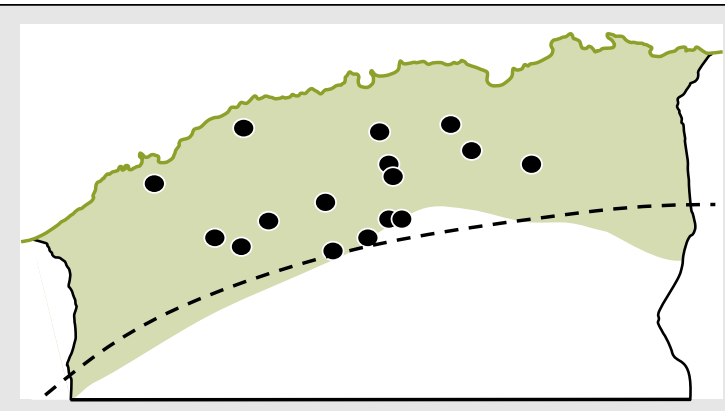

A. Hordeum murinum

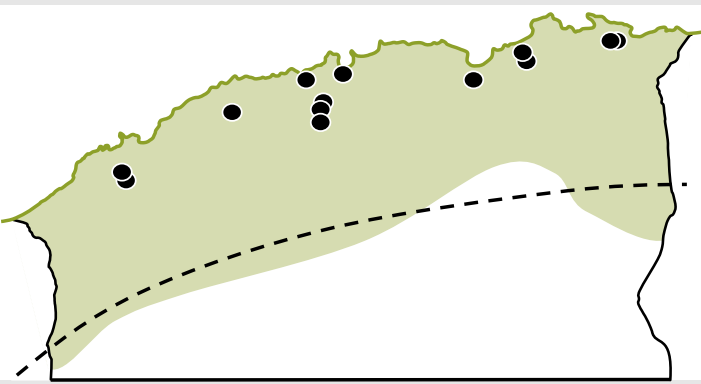

C. Dactylis glomerata

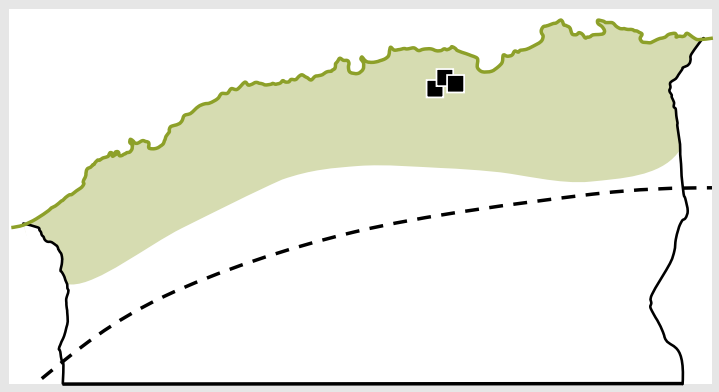

B. Aegilops neglecta

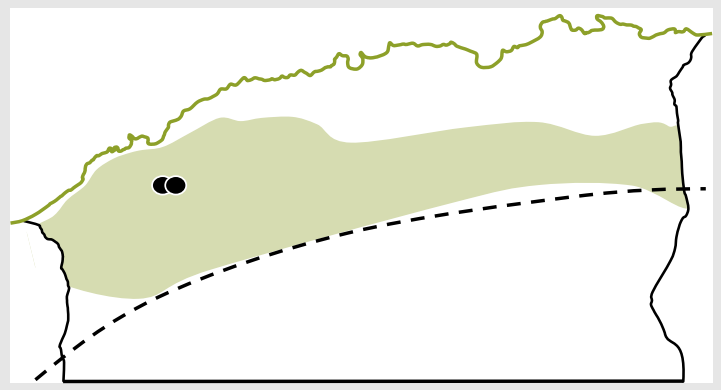

D. Lygeum spartum

Figure 2. Aire de distribution géographique de quatre Poaceae polyploïdes en Algérie tellienne.

Figure 2. Geographical distribution of four polyploid species of Poaceae in North Algeria.

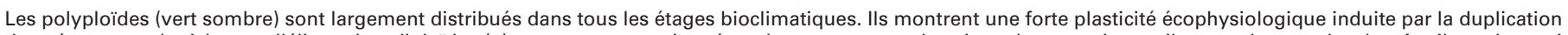

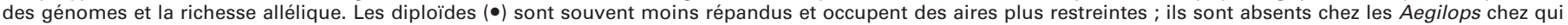

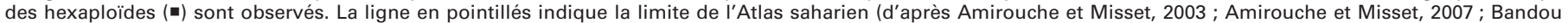
2005 ; Bandou et al., 2009 ; Benmansour et Harche, 2001).

et se répartissent largement depuis la marge littorale, au nord, jusqu'à la bordure saharienne, au sud (figure $2 A$ ). La diversité génétique est largement répartie à l'intérieur des populations dont le faible degré de différenciation suppose l'existence d'un flux de gènes appréciable entre elles.

En revanche, les populations diploïdes de la sous-espèce glaucum, sont nettement plus différenciées et sont caractérisées par un isolement relatif, plus continental, principalement dans les étages bioclimatiques arides et semi-arides de la bordure saharienne (figure 2A). Cette localisation marginale est associée à une tolérance vis-à-vis des basses températures hivernales, de la sécheresse et de la salinité. Dans ces populations 2x, la diversité interpopulations, notamment des protéines de réserve (hordéines), s'accroît régulièrement en fonction du gradient d'aridité (Amirouche et Misset, 2003).

Ainsi, la distribution spatiale en Algérie des orges 2x (glaucum) et 4x (leporinum) plaiderait en faveur de processus adaptatifs et évolutifs encore en cours, particulièrement au sein des populations diplö̈- des "endémiques " des zones arides et semi-arides.

\section{Egilopes (Aegilops)}

Les Aegilops sont représentés en Algérie par des espèces tétraploïdes, dont A. triuncialis L., A. geniculata Roth., A. biuncialis Vis., A. neglecta Req. et $A$. ventricosa Tausch. Certaines espèces sont largement répandues et couvrent divers étages bioclimatiques et milieux écologiques. C'est le cas d'A. geniculata $(2 \mathrm{n}=4 \mathrm{x}=28)$ dont l'étude morphologique (Bandou, 2005), a permis de mettre en évidence une grande variabilité avec des phénotypes liés aux conditions de haute altitude (Chélia, Aurès, Djurdjura) et des phénotypes liés aux conditions de sécheresse (Hauts Plateaux). Dans les autres stations de l'Atlas tellien, où les conditions bioclimatiques sont fluctuantes, la diversité intrapopulation est importante. Le polymorphisme des gluténines a permis de séparer nettement les espèces étudiées : A. geniculata, $A$. neglecta, $A$. triuncialis et A. ventricosa (Bandou et al., 2009). Chez $A$. neglecta, des profils protéiques typiques d'hexaploïdes ( $2 n=6 x=42)$ ont été observés puis confirmés en cytologie. Ces cytotypes, nouvellement signalés en Algérie, sont localisés dans le Djurdjura (figure 2B).

\section{Dactyle (Dactylis)}

Le dactyle est un genre monotypique représenté par une espèce vivace importante par sa haute valeur pastorale et fourragère : Dactylis glomerata L. C'est un modèle typique de polyploïdie et d'évolution (Stebbins et Zohary, 1959; Lumaret, 1988 ; Soltis et Soltis, 1993).

En Algérie, les populations de cette espèce sont très largement répandues et montrent une plasticité phénotypique et une amplitude écologique considérables, s'exprimant par des habitats très diversifiés: falaises maritimes, plaines du littoral et du Sahel, collines et gorges de l'Atlas tellien, pelouses d'altitude, steppes des Hauts Plateaux et Atlas saharien.

L'existence en Algérie septentrionale des deux niveaux de ploïdie est confirmée (Amirouche, 2005) et leur statut taxonomique précisé, avec des sous-espèces diploïdes - D. glomerata ssp. mairei 
Stebbins et Zohary et ssp. santai Stebbins et Zohary - et des sous-espèces tétraploïdes - D. glomerata ssp. hispanica (Roth) Koch. et ssp. marina (Borrill) Greuter (Amirouche et Misset, 2007).

Finalement, les diploïdes sont bien plus abondants que supposé antérieurement (figure 2C) et ont un comportement méiotique fortement relié aux conditions écogéographiques.

La sous-espèce mairei, localisée essentiellement dans des niches écologiques humides des secteurs de la KabylieNumidie, présente une méiose régulière, tandis que la sous-espèce santai se singularise par un taux élevé de bivalents droits, d'univalents et de chromosomes B. Cette dernière est plus largement répandue dans les étages subhumides et semi-arides des secteurs algérois et pranais (figure 2C).

En périphérie de l'aire de distribution et dans les zones de transition, le contact diploïdes/diploïdes et diploïdes/tétraploïdes entraînerait des flux de gènes et l'émergence de génotypes différents (Amirouche, 2005 ; Amirouche et Misset, 2007). Le taux d'hétérozygotie élevé mis en évidence par les marqueurs enzymatiques, la présence significative d'univalents, ainsi que l'observation d'individus triplö̈des, appuient l'existence de tels échanges géniques.

\section{Sparte (Lygeum)}

Un autre exemple de graminée pérenne monotypique est celui du sparte Lygeum spartum L., une des composantes des steppes nord-africaines, potentiellement intéressante pour l'industrie papetière (Harche et al., 1990). Les premières investigations cytotaxonomiques (Benmansour et Kaid-Harche, 2001) ont confirmé la prépondérance en Algérie des cytotypes polyploïdes $(2 \mathrm{n}=40)$, depuis le littoral et les Hauts Plateaux du secteur oranais jusqu'aux limites orientales du secteur constantinois. Elles ont permis de signaler, pour la première fois en Algérie, la présence de cytotypes à $2 \mathrm{n}=16$, structurés en petites populations isolées dans le Nord-Ouest des Hauts Plateaux du Sud oranais (figure 2D). Ces populations seraient diploïdes $(2 n=2 x=16)$. Elles possèdent des infrutescences vigoureuses dont les lemmes ont des parties libres plus courtes que celles des polyploïdes. Ces derniers se distinguent par des infrutescences lâches et moins vigoureuses et une capacité reproductrice fortement perturbée (Djabeur et al., 2008).
La répartition très restreinte des diploïdes, par rapport aux polyploïdes, suggère que L. spartum est un complexe "mature" (Stebbins, 1971). Il s'agira d'établir avec précision les limites de l'aire de répartition des diplö̈des et de préciser leurs liens avec les polyplö̈des.

\section{Légumineuses (Fabaceae)}

Dans la flore algérienne, les Fabaceae sont représentées par environ 360 espèces et sous-espèces, dont $41 \%$ rares à très rares et $13 \%$ endémiques au sens large (d'après Quézel et Santa, 1962). Du fait de son importance fourragère et pastorale, cette famille a bénéficié d'une attention particulière de la part de différentes équipes de recherche.

Divers travaux sur les genres Medicago, Trifolium, Scorpiurus, Hedysarum et Onobrychis (Abdelguerfi et Laouar, 1999) ont montré une corrélation positive entre les paramètres biogéographiques, particulièrement l'altitude et la pluviosité, et les traits biologiques liés à la croissance et à la reproduction - floraison, inflorescences, gousses et graines.

Chez les luzernes, précisément dans le complexe Medicago ciliaris-intertexta, cette variabilité soulève aussi des questions d'ordre taxonomique. Les deux espèces, $M$. ciliaris (L.) All. et $M$. intertexta (L.) Mill., ont une répartition à tendance allopatrique. La première, dans le NordOuest, montre une tolérance vis-à-vis de la sécheresse; la seconde se rencontre essentiellement dans les régions humides du Nord-Est (Laouar et al., 2000 ; Abdelguerfi-Laouar et al., 2003). Dans les situations de sympatrie, des "populations particulières "se différencient par la forme des épines et la pilosité des gousses. Leur isolement par rapport aux deux autres taxons, ciliaris et intertexta, est confirmé par les marqueurs microsatellites et, sans qu'il soit encore possible d'en préciser une nature hybride, leur "originalité taxonomique " est mise en exergue par leur capacité symbiotique spécifique avec Sinorhizobium meliloti (AbdelguerfiLaouar et al., 2006).

On citera aussi l'exemple de Lupinus angustifolius L., chez qui une tendance à une différenciation écotypique s'exprime par des critères structuraux et biochimiques des graines (Aïnouche, 1998). Dans les stations arides et semi-arides du secteur oranais, les phénotypes à graines réticulées "reticulatus" sont rares et seraient en voie de disparition, tandis que les phénotypes à " grosses graines " ne se rencontrent que dans les milieux anthropisés des secteurs humides et subhumides du Centre-Est du pays. En revanche, chez L. micranthus Guss., la variabilité interpopulations, continue, est particulièrement élevée dans les zones de transition bioclimatique.

Ce modèle de variation a été également observé dans le genre Hedysarum. Chez $H$. coronarium L., Issolah et Khalfallah (2007) ont montré l'influence de l'altitude et de la pluviosité sur la variabilité morphologique et phénologique de populations du Nord-Est de l'Algérie. Un fait remarquable au sein des populations situées à la périphérie de l'aire de distribution, est la coexistence de deux cytotypes : l'un attendu, $(2 \mathrm{n}=16 ; \mathrm{x}=8)$, l'autre nouveau $(2 \mathrm{n}=18 ; \mathrm{x}=9)$ et qui présente une paire additionnelle d'autosomes (Issolah et al., 2006).

Cette variation du nombre chromosomique par dysploïdie, associée aux conditions écologiques locales, a été signalée chez d'autres espèces algériennes de légumineuses.

\section{Scilles automnales (Hyacinthaceae)}

Les Monocotylédones bulbeuses et la famille des Hyacinthaceae en particulier, sont des plantes qui présentent de multiples intérêts génétiques, évolutifs et écologiques. Du point de vue biochimique et de la pharmacopée, certaines espèces sont réputées pour leur production abondante en triterpènes, dont des hétérosides scillirosides à activité cardiotonique et raticide, tandis que d'autres renferment des flavonols, des kaempferols et des quercetols (Krenn et al., 2001).

Les Hyacinthaceae constituent une bonne illustration de la nécessité de réviser et de mettre à jour la flore de l'Algérie. En effet, plusieurs genres de l'ancienne famille des Liliaceae ont été répartis en plusieurs familles dans l'ordre des asparagales, selon la nouvelle classification phylogénétique des angiospermes (APG II, 2003). Dans la flore de Quézel et Santa (1962), ce genre est représenté par sept espèces dont quatre automnales, endémiques de 


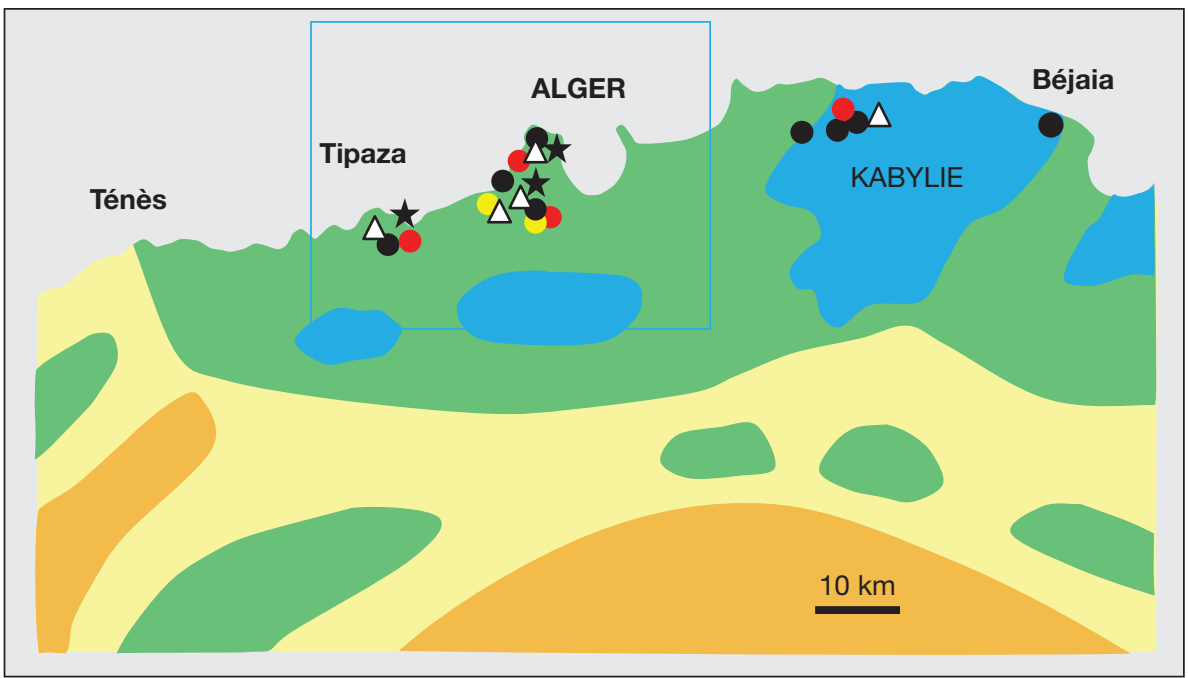

Figure 3. Localisation de quelques populations de scilles automnales.

Figure 3. Localization of some populations of autumnal squills.

On remarquera la concentration de taxons endémiques dans une aire réduite du littoral algérois située en zone de transition biogéographique (les couleurs des zones correspondent aux étages bioclimatiques de la figure 1) (modifié d'après Hamouche et al., 2006).

Prospero autumnale $(\bullet 2 n=14 ; \cdot 2 n=28 ; \bullet 2 n=42), P$. obtusifolium $(\star 2 n=8)$, Barnardia numidica $(\Delta 2 n=18)$

l'Afrique du Nord et du Centre-Est de l'Algérie. Des inventaires caryologiques effectués sur une aire relativement restreinte de l'Algérois (figure 3) ont révélé une concentration exceptionnelle de cytotypes différents (Hamouche et al., 2006). Selon la nomenclature mise à jour dans ce groupe par Speta (1998) et acceptée pour la flore d'Afrique du Nord, ces cytotypes correspondraient à Prospero autumnale (L.) Speta (= Scilla autumnalis $\mathrm{L}.)-2 \mathrm{n}=14,2 \mathrm{n}=28,2 \mathrm{n}=42$; Prospero obtusifolium (Poir.) Speta (= Scilla obtusifolia Poir.) - 2n=8; Barnardia numidica (Poir.) Speta (= Scilla numidica Poir.) $-2 n=18$.

Cependant, des ambiguités taxonomiques persistent. Elles sont dues à l'existence de formes intermédiaires fréquentes dans les forêts du littoral algérois, véritable carrefour entre les secteurs biogéographiques oranais et kabyle. Elles résulteraient d'hybridations favorisées par la situation de sympatrie impliquant les taxons en présence.

\section{Conclusions et perspectives}

Toutes ces observations indiquent qu'en Algérie, des conditions bioclimatiques très variées joueraient un rôle majeur sur la structuration des populations et des espèces. Elles révèlent aussi l'ampleur de la polyploïdie comme manifestation de la diversité génétique.

Certaines populations, surtout diploïdes, ont tendance à s'isoler en petites populations en périphérie de l'aire de distribution - comme c'est le cas, sur les Hauts Plateaux, d'Hordeum murinum ssp. glaucum (2x) et de Lygeum spartum (2x) ou encore, dans les biotopes frais de l'Atlas tellien, des sous-espèces diploïdes de Dactylis glomerata. Ces populations sont remarquables de par leur taux de différenciation interpopulations élevé. Les fluctuations des conditions écologiques associées à leur isolement favoriseraient, par les effets de la sélection et de la dérive génétique, l'émergence de génotypes à fort potentiel évolutif.

On reconnait aujourd'hui que de telles populations en limite d'aire sont plus résistantes et ont un potentiel adaptatif plus élevé face aux changements environnementaux que les populations centrales (Volis et al., 1998 ; Araújo, 2002). Elles représentent de ce fait une valeur comme réservoir de gènes d'intérêt utilisables en amélioration et sont à privilégier dans l'optique de la conservation.

Dans les zones de transition bioclimatique, les populations sont souvent caractérisées par une diversité génétique importante. Ces zones méritent une attention particulière dans les efforts de conservation de la biodiversité, car elles peuvent constituer des centres de spéciation suite à des micro-isolements. Selon Stebbins et Major (1965), les localités où la spéciation a été la plus intense, en Californie, sont celles de plus grande variabilité climatique et édaphique et, surtout, celles qui occupent une position de transition.

La connaissance des limites d'aires de répartition des espèces et des polyploïdes et l'estimation des flux de gènes le long du gradient écologique s'avèrent donc déterminants comme sources d'indications sur le potentiel évolutif des programmes d'évaluation et de conservation de la biodiversité.

Les événements cytogénétiques - polyploïdie, chromosomes B, méioses irrégulières... -, observés dans les modèles exposés (Poaceae, Fabaceae, Hyacinthaceae) ou en cours d'études (Alliaceae), semblent associés aux paramètres écogéographiques et soulèvent des questions d'ordre évolutif. La connaissance de l'origine de la polyploïdie peut aussi apporter des éléments explicatifs sur les conséquences des hybridations dans les zones de contact (Schierenbeck et Aïnouche, 2006).

Néanmoins, la formation des complexes spécifiques polyploïdes favorise l'émergence de continuum morphologiques générant des interrogations taxonomiques. Une mise à niveau sur les plans taxonomique et nomenclatural de la flore d'Algérie doit être le point de repère pour l'évaluation et la conservation de la biodiversité.

\section{Remerciements}

Les auteurs tiennent à remercier Nabila Amirouche et Houria Hadj-Arab qui ont permis d'améliorer le manuscrit, ainsi que Houda Bandou, Yasmina Hamouche (université des sciences et de la technologie Houari Boumediene [USTHB], Alger), Meriem Harche-Kaid (université d'Oran), Nadra Khalfallah (université de Constantine), Nadia Bouguedoura (USTHB, Alger) et Aïssa Abdelguerfi (Institut national agronomique) pour leur appui bibliographique.

\section{Références}

Abdelguerfi A, Laouar M. Autoécologie et variabilité de quelques légumineuses d'intérêt fourrager et/ou pastoral : possibilités de valorisation en région méditerranéenne. Pastagens e Forragens $1999 ; 20: 81-112$. 
Abdelguerfi-Laouar M, Abdelguerfi A, Bouznad $Z$, Guitonneau GG. Autoécologie du complexe d'espèces Medicago ciliaris - M. intertexta en Algérie. Acta Bot Gallica 2003 ; 150 : 253-65.

Abdelguerfi-Laouar M, Huguet T, Abdelguerfi $A$ et al. Structuration de la diversité des légumi neuses et de leurs Rhizobium associés : section des intertextae (Medicago). In: Actes des $\mathrm{X}^{\mathrm{e}}$ journées scientifiques de I'Agence universitaire de la Francophonie, Quelles Biotechnologies pour une agriculture durable ? Constantine, 811 mai 2006. Constantine (Algérie) : imprimerie de l'université Mentouri, 2006.

Aïnouche A. Diversité et évolution du genre Lupinus L. (Fabaceae). Thèse de doctorat, université de Rennes 1, 1998.

Alcaraz C. Recherches géobotaniques sur la végétation de l'ouest algérien. Bull Soc Hist Nat Afrique du Nord 1976; 67 : 19-36.

Amirouche N. Diversité génétique de populations diploïdes et tétraploïdes de Dactylis glomerata L. (Poaceae). Recherche Agronomique $2005 ; 16: 53-63$

Amirouche N, Misset MT. Morphological variation and distribution of cytotypes in the diploid-tetraploid complex of the genus Dactylis L. (Poaceae) from Algeria. Plant Syst and Evol $2007 ; 264: 157-74$.

Amirouche R, Misset MT. Hordein polymorphism in diploid and tetraploid Mediterranean populations of the Hordeum murinum L. complex. Plant Syst and Evol 2003; 242 : 83-99.

APG II. An update of the Angiosperm Phylogeny Group classification for the orders and families of flowering plants. Bot J Linn Soc $2003 ; 141: 399-436$

Araújo BM. Biodiversity hotspots and zones of ecological transition. Conserv Biol 2002; 16 : 1662-3.

Bandou H. Les populations d'Aegilops: diversité morphologique et diversité biochimique. Recherche Agronomique 2005 ; 16 : 28-35.

Bandou H, Rodriguez-Quijano M, Carrillo JM, Branlard G, Zaharieva M, Monneveux P. Morphological and genetic variation in Aegilops geniculata from Algeria. Plant Syst and Evol 2009 ; 277 : 85-97.

Battandier JA, Trabut L. Flore d'Alger et catalogue des plantes d'Algérie. Alger: Adolphe Jourdan libraire-éditeur, 1884.

Battandier JA, Trabut L. Flore analytique et synoptique de I'Algérie et de la Tunisie. Alger: Vve Giralt imprimeur-éditeur, 1902.

Benmansour N, Kaid-Harche M. Étude caryologique de deux populations de Lygeum spartum L. de l'Ouest algérien. Bocconea 2001; 13 371-6.
Desfontaines R. Flora Atlantica, sive, Historia plantarum, quae in Atlante, agro Tunetano et Algeriensi crescunt. Tomes 1 et 2. Paris : Apud Blanchon, 1798-1799.

Djabeur A, Kaid-Harche M, Catesson AM. Structure des infrutescences et capacité fructifère de deux cytotypes de Lygeum spartum L. de l'ouest algérien. Ecologia mediterranea $2008 ; 34: 5-12$

Dobignard A. Index synonymique et bibliographique de la flore d'Afrique du Nord. Genève: Conservatoire et jardins botaniques de la ville de Genève, 2009 (sous presse. http://www.ville-ge.ch/musinfo/bd/cjb/ africa/recherche.php).

Hamouche $\mathrm{Y}$, Amirouche $\mathrm{R}$, Amirouche N. Notes taxonomiques et caryologiques de deux espèces du genre Scilla (Hyacinthacées) de I'ouest algérois. Ann Inst Nat Agro 2006; 27 : 95-105.

Harche M, Chadli R, Catesson AM. Diversity of cellulose microfibril arrangement in the cell walls of Lygeum spartum leaves. Annals of Botany $1990 ; 65$ : 79-86.

Hoekstra JM, Boucher TM, Ricketts TM, Roberts C. Confronting a biome crisis: global disparities of habitat loss and protection. Ecol Lett $2005 ; 8$ : 23-9.

Issolah R, Benhizia H, Khalfallah N. Karyotype variation within some natural populations of sulla (Hedysarum coronarium L., Fabaceae) in Algeria. Genet Resour Crop Ev 2006; 53: 1653-64.

Issolah $\mathrm{R}$, Khalfallah $\mathrm{N}$. Analysis of the morpho-physiological variation within some Algerian populations of sulla (Hedysarum coronarium L., Fabaceae). Journal of Biological Sciences 2007 ; 7 : 1082-91.

Krenn L, Kopp B, Speta F, Kubelka W. Chemotaxonomical investigation of the genus Charybdis Speta (Urgineoideae, Hyacinthaceae). Stapfia $2001 ; 75: 101-20$.

Laouar M, Abdelguerfi A, Kouchi K. Etude du complexe d'espèces Medicago ciliaris - $M$. intertexta. Variabilité morphologique et phénologique. Ann Inst Nat Agro 2000; 21 : 51-70.

Lumaret R. Cytology, genetics and evolution in the genus Dactylis. Crit Rev Plant Sci 1988; 7 : 55-91.

Maire R. Flore de l'Afrique du Nord. Volumes 1-16. Paris: Paul Le Chevalier, 1955-1987.

Médail F, Quézel P. Conséquences écologiques possibles des changements climatiques sur la flore et la végétation du bassin méditerranéen. Bocconea 2005: 16 : 397-422.

Médail F, Quézel P. Hot-spots analysis for conservation of plant biodiversity in the Mediterranean basin. Ann Missouri Bot Gard 1997; $84: 112-27$.
Médiouni K. Élaboration d'un bilan et d'une stratégie nationale de développement durable de la diversité biologique. Projet ALG/97/G31. FEM/PNUD. Tome I à VIII. Alger: éditions du ministère de l'Aménagement du Territoire et de l'Environnement, 2004

Ohba H, Amirouche R. Observation of the Flora of Tadmait and Tidikelt, Central Sahara, Algeria. Journal of Japanese Botany 2003; 78 : 104-11.

Poiret JML. Voyage en Barbarie ou Lettres Ecrites de l'ancienne Numidie pendant les années 1785 et 1786. Seconde partie. Recherches sur l'Histoire Naturelle de la Numidie. Paris : JBF née de la Rochelle, 1789.

Quézel P, Médail F. Ecologie et biogéographie des forêts du bassin méditerranéen. Paris: Elsevier, 2003.

Quézel P, Santa S. Nouvelle flore de l'Algérie et des régions désertiques méridionales. Tomes 1 et 2. Paris: CNRS, 1962-1963.

Sala OE, Chapin FS, Armesto JJ, et al. Global biodiversity scenarios for the year 2100. Science $2000 ; 287$ : 1770-4.

Schierenbeck KA, Aïnouche ML. The role of evolutionary genetics in studies of plant invasion. In : Cadotte M, Mc Mahon SM, Fukami T, eds. Conceptual Ecology and Invasion Biology: Reciprocal approaches to Nature. Heidelberg: Springer, 2006.

Soltis DE, Soltis PS. Molecular data and the dynamic nature of polyploidy. Crit Rev Plant Sci $1993 ; 12: 243-73$.

Speta F. Systematische Analyse der Gattung Scilla L. s. I. (Hyacinthaceae). Phyton (Horn Austria) $1998 ; 38: 1-141$.

Stebbins GL. Chromosomal evolution in higher plants. London: Edward Arnold Publishers, 1971.

Stebbins GL, Major J. Endemism and speciation in the California Flora. Ecol Monogr $1965 ; 35: 1-35$

Stebbins GL, Zohary D. Cytogenetic and evolutionary studies in the genus Dactylis. I Morphology, distribution and inter relationships of the diploid subspecies. University of California Publications in Botany 1959 ; 31 : 1-40.

Stewart P. Un nouveau climagramme pour I'Algérie et son application au barrage vert. Bull Soc Hist Nat Afrique du Nord 1974; 65 : 239-48.

Véla E, Benhouhou S. Évaluation d'un nouveau point chaud de biodiversité végétale dans le Bassin méditerranéen (Afrique du Nord). $C R$ Biol 2007 ; 330 : 589-605.

Volis S, Mendlinger S, Olsvig-Whittaker L, Safriel UN, Orlovsky N. Phenotypic variation and stress resistance in core and peripheral populations of Hordeum spontaneum. Biodivers Conser $1998 ; 7: 799-813$. 